\title{
CLINICAL PROFILE OF HYMENOPTERA STING IN A TERTIARY CARE HOSPITAL IN HIMACHAL PRADESH
}

\section{Pratibha Himral ${ }^{1}$}

${ }_{1}^{1}$ Assistant Professor, Department of General Medicine, Dr. Rajendra Prasad Medical College, Kangra, Himachal Pradesh, India.

\section{ABSTRACT}

\section{BACKGROUND}

Hymenoptera stings are common public health hazards worldwide. Clinical spectrum of venom allergy varies from local reaction to life-threatening and unusual delayed reaction which may be fatal.

\section{MATERIALS AND METHODS}

This retrospective study was done to assess the clinical profile and outcome of hymenoptera sting in a tertiary care hospital in Himachal Pradesh. 33 patients admitted in medicine ward were analysed.

\section{RESULTS}

Incidence of hymenoptera sting was more common among females (57.6\%) as compared to males (42.4\%). Majority of the wasp and bee stings were observed in the months of October to December (50\%). Local inflammatory reactions were present in all the patients with wasp and bee sting. Most common complication was hepatitis (39.4\%) followed by hypertension $27.3 \%$ ) and acute renal injury (15.2\%). Mortality rate in our study was $12.1 \%$. Local reactions were mild and resolved spontaneously.

\section{CONCLUSION}

Wasp and bee stings result in mild reactions to life-threatening complications, which may be delayed in onset. Therefore, early anticipation of these complications by the treating physician is needed for the management of the patient.

\section{KEY WORDS}

Hymenoptera Sting, Hepatotoxicity, Acute Kidney Injury.

HOW TO CITE THIS ARTICLE: Himral P. Clinical profile of hymenoptera sting in a tertiary care hospital in Himachal Pradesh. J. Evolution Med. Dent. Sci. 2018;7(43):4675-4677, DOI: 10.14260/jemds/2018/1042

\section{BACKGROUND}

Hymenoptera stings are common worldwide, especially in countries with a predominantly moderate climate. In this environment, these stinging insects are present for larger part of the year and around $56 \%$ to $94 \%$ of the population is stung at least once in their lifetime. ${ }^{1}$ All the stinging insects belong to the order Hymenoptera of the class Insecta. Medically important hymenopterans are Apidea (Honey bee and bumble bee), Vespidae (Wasp, hornets and yellow jackets) and Formicidae (Ants). The venomous stinger of hymenoptera evolves from their ovipositor that has lost egglaying function and modified for stinging and delivering the venom to their victims. Bee loses its barbed stinger after the sting, whereas a single wasp is able to sting multiple times because the stinger is not detached from the body after the sting. ${ }^{2}$

These stinging events occur when a single insect is disturbed while searching for food or in large swarm when insects respond to a human intruder as a threat to their colony, usually in late summer or early fall. ${ }^{3}$ These insects preferentially sting in the head and neck area. The amount of venom released during a wasp sting is upto $3 \mu \mathrm{gm}$ as compared to $140 \mu \mathrm{gm}$ released per bee sting.

'Financial or Other Competing Interest': None.

Submission 10-09-2018, Peer Review 04-09-2018,

Acceptance 11-10-2018, Published 22-10-2018.

Corresponding Author:

Pratibha Himral

Dr. Rajendra Prasad Medical College,

Tanda District, Kangra,

Himachal Pradesh, India.

E-mail: shah.pratibha24@gmail.com

DOI: $10.14260 /$ jemds $/ 2018 / 1042$

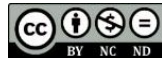

The components of venom include enzymes such as phospholipase A1 and A2 and hyaluronidase, peptides such as melittin, amines such as histamine, serotonins and catecholamines and others such as mastoparan, apamine, acetylcholine and antigen 5.4,5 These components have inflammatory, direct and indirect cytotoxic (Renal, hepatic and membrane), haemolytic, vasoactive, neurotoxic and cellular anti-membrane properties resulting in local as well as systemic manifestations. ${ }^{6}$

Reactions to hymenoptera envenomation are classified into normal local reaction, large local reaction, systemic anaphylactic reactions and delayed unusual reactions. After the sting patient may develop minor local reaction of erythema, oedema and pain at the site of sting which is selflimiting and resolves spontaneously within 24 hours. Large local reaction is defined as an area of induration exceeding a diameter of $10 \mathrm{~cm}$ which lasts longer than 24 hours and then subsides. Prevalence of systemic anaphylactic reaction is $0.3 \%-7.5 \%$. These reactions are usually apparent within 15 minutes of sting and vary in intensity from mild (Urticaria, pruritis, angioedema, vomiting and diarrhoea) to lifethreatening reactions (Wheeze, cough, dyspnoea and bronchoconstriction). The unusual delayed reactions are the rare clinical presentations of hymenoptera sting and include serum sickness, vasculitis, thrombocytopenic purpura, renal, neurological, cardiovascular and skin manifestations. Incidence of mortality in wasp and bee sting is low and ranges from 0.03 to 0.48 per 1000000 inhabitants per year. $2,4,5,7$

\section{MATERIALS AND METHODS}

We, in this retrospective descriptive study, analysed 33 cases of multiple wasp and bee stings above 18 years of age 
admitted in medicine ward of Dr. Rajendra Prasad Medical College, Tanda, Himachal Pradesh from July 2015 to June 2016. Data was analysed with respect to age, sex, clinical manifestations, duration of hospitalisation, complications and outcome.

\section{RESULTS}

In this retrospective descriptive study, we reviewed 33 patients of wasp and bee sting who were admitted in our hospital from July 2015 to June 2016 retrospectively. The youngest patient was of 18 years, whereas the age of the oldest patient was of 92 years. The mean age was $44.6 \pm 18.1$ years. Most of the patients were more than 45 years of age (51.4\%). Incidence of hymenoptera sting was more common among females (57.6\%) as compared to males (42.4\%). The male-to-female ratio was 0.74 in the present study. Most of the wasp and bee stings were observed in the months of October to December (50\%) followed by April to June (34.4\%).

All the patients had local inflammatory reactions e.g. redness, pain, swelling and burning sensation at the site of sting. Myalgias were observed in $33.3 \%$ cases and body swelling in $30.3 \%$ cases. $8(24.2 \%)$ patients had dyspnoea. Vomiting and oliguria was present in 5 patients each (15.2\%), headache $(12.1 \%)$, altered sensorium $(6.06 \%)$ and dysphagia (6.06\%). $65.6 \%$ of the patients reached hospital within 6 hours from the time of sting. Most common site of sting was head and face (57.6\%). Icterus was present in 3 patients (9.09\%). The most common complication was hepatitis (39.4\%) followed by hypertension (27.3\%) and AKI (15.2\%). Out of 5 patients with AKI, 2 patients required haemodialysis, but one patient died. The hospitalisation time varied from 30 minutes to 6 days with a mean duration of hospitalisation of 3 days in the present study. $84 \%$ patients were discharged from hospital after the treatment, whereas one patient left against medical advice. 4 patients died due to hymenopteran stings in our study and in hospital mortality was $12.1 \%$. Two patients $(50 \%)$ had multi-organ dysfunction, one patient died of anaphylactic shock and another one died of nonanaphylactic shock.

\section{DISCUSSION}

Of total 33 cases included in our study, most of the patients belonged to the age group of 18 - 45 years. The mean age of the patients was $44.6 \pm 18.1$ years. In the study done by Witharana et al, ${ }^{8}$ majority of the patients were from $20-60$ years of age and mean age was $42 \pm 15.1$ years. 8 people in this age group are at a risk of wasp and bee stings, because this is the main working age group. Majority of people in our state live in rural setup and are agriculturist. They go out for work in early morning hours and continue late till dusk. This also increases the risk of sting due to the poor visibility.

A female preponderance was observed in our study and male-to-female ratio was 0.74 . This increased incidence of wasp and bee sting in females may be due to the fact that females go to the forest for grass cutting and wood collection and also work for longer periods in a day in the fields in our state. Second reason could be the colourful clothing worn by females often attract the bee and wasp. Similar female preponderance was also observed in other studies.9,10

Maximum number of wasp and bee stings in our study were observed in autumn season. Wasps become pests in summer and autumn and sting if it is accidentally stepped on, swatted or disturbed while searching food resulting in significantly higher monthly sting rates in late summer and autumn. ${ }^{11}$ Ghimire et al had reported higher incidence of hymenopteran stings during months from July to September. ${ }^{9}$

The most common clinical manifestations observed in our study included local inflammatory reaction (Redness, pain, swelling and burning sensation) at site of sting, myalgias, vomiting, oliguria, headache, altered sensorium, dysphagia and jaundice. Local inflammatory reactions, dizziness, nausea, vomiting, haematuria, decreased urinary output, loss of consciousness, convulsions, hypotension, arrhythmia and acute respiratory distress syndrome were common clinical manifestations observed by Cuihong Xie et al and Ghimire et al.9,12

Serum bilirubin and transaminase levels were done in all 33 patients. Transaminase levels were increased in $48.5 \%$ patients. (45.5\%) patients had increase in AST (Aspartate aminotransferase) levels more than ALT (Alanine aminotransferase) level, whereas one patient had increase in ALT levels more than AST levels. Total bilirubin levels were elevated in $27.3 \%$. Tsai et al had reported a case of markedly elevated aminotransferase levels after hornet sting. Maximum level of ALT and AST were $3156 \mathrm{U} / \mathrm{L}$ and $3006 \mathrm{U} / \mathrm{L}$ respectively. ${ }^{13}$ In our study, two patients had markedly elevated levels of AST and ALT (4728 U/L and $1612 \mathrm{U} / \mathrm{L}$, $2584 \mathrm{U} / \mathrm{L}$ and $1849 \mathrm{U} / \mathrm{L}$ ). Mechanism responsible for hepatic dysfunction from hornet venom was studied by Barr-Nea et al in rat liver after repeated envenomation with hornet venom sac extract. Foci of hepatocyte necrosis, fat infiltration and increased alkaline phosphatase activity was observed by light microscopy and histochemistry analysis. Under the electron microscope, destruction of mitochondria in hepatocyte and damage to membrane of bile canaliculi were observed, which resulted in both hepatocellular damage and cholestasis in rat model. ${ }^{14}$ In another animal model study, Neuman et al found that hepatotoxicity associated with venom sac extract was in proportion to the dose of toxin used and the liver damage was of cholangiocellular type. ${ }^{15}$

Acute renal injury due to hymenopteran sting was found in $15.2 \%$ of cases in our study. All three patients with rhabdomyolysis developed AKI. Two patients required haemodialysis and one of them died. The mechanisms of AKI are multi-factorial in wasp and bee stings. These mechanisms include pigment induced ATN, AIN and rarely acute cortical necrosis and thrombotic microangiopathy. Rhabdomyolysis and haemolysis are caused by melittin and phospholipase present in wasp venom. Myoglobin is freely filtered in glomeruli and leads to AKI by toxic effects on tubular epithelial cells through intralobular cast nephropathy and pigment nephropathy. Myoglobin is also potent inhibitor of nitric oxide and trigger intrarenal vasoconstriction and ischaemia in patients with dehydration and acidic urine. ${ }^{16,17,18}$

In our study, 5 patients developed liver dysfunction as well as renal dysfunction. Multi-organ dysfunction due to single wasp sting and massive wasp stings have been reported.19,20 Mean duration of hospitalisation in our study was three days.

Mortality rate in our study was $12.1 \%$. Cuihong Xie et al had reported a death rate of $5.1 \%$ in their study. 12 There was no death due to the wasp and bee sting in studies done by 
Ghimire et al and Witharana et al.8,9 Majority of deaths related to wasp and bee sting are the direct results of immediate hypersensitivity reaction mediated by IgE and resulting in anaphylaxis. Mortality rate due to mass envenomation ranges from 15 - 25\%. Death from multiple stings results from three major mechanisms: direct venom toxicity, intravascular haemolysis mediated by melittin and profound hypotension resulting from massive histamine release.

\section{CONCLUSION}

Wasp and bee stings occur frequently. Clinical manifestations of hymenoptera sting vary from mild reactions to lifethreatening complications, which may be delayed in onset. Therefore, early anticipation of these complications is needed for the management of the patients.

\section{REFERENCES}

[1] Antonicelli L, Bilo MB, Bonifazi F. Epidemiology of hymenoptera allergy. Curr Opin Allergy Clin Immunol 2002;2(4):341-6.

[2] Fitzgerald KT, Flood AA. Hymenoptera stings. Clin Tech Small Anim Pract 2006;21(4):194-204.

[3] Edwards R. Social Wasps. Their biology and control. East Grinstead, United Kingdom: Rentokil Ltd., 1980.

[4] Bilo BM, Rueff $F$, Mosbech $\mathrm{H}$, et al. Diagnosis of Hymenoptera venom allergy. Allergy 2005;60(11):1339-49.

[5] Przybilla B, Rueff F. Insect stings: clinical features and management. Dtsch Arztebl Int 2012;109(13):238-48.

[6] Singh Y, Joshi SC, Saxena SR, et al. Acute renal failure: a fatal complication following multiple hornet stings. Int J Health Allied Sci 2014;3(1):56-9.

[7] Krishana MT, Ewan PW, Diwakar L, et al. Diagnosis and management of hymenoptera venom allergy: British Society for Allergy and Clinical Immunology (BSACI) guidelines. Clinical \& Experimental Allergy 2011;41(9):1201-20.

[8] Witharana EWRA, Wijesinghe SKJ, Pradeepa KSM, et al. Bee and wasp stings in Deniyaya: a series of 322 cases. Ceylon Medical Journal 2015;60(1):5-9.
[9] Ghimire M, Pahari B, Paudel N, et al. Hymenoptera stings: a study of clinical profile, complication and outcome from a teaching hospital of central Nepal. Journal of College of Medical Sciences-Nepal 2013;9(3):17-24.

[10] Goel S, Gupta H, Mazta S. Epidemiological profile of sting cases admitted at a 50 bedded Community Health Centre of Himachal Pradesh, India. The Internet Journal of Health 2007;7(1).

[11] Bischof R0. Seasonal incidence of insect stings: autumn 'yellow jacket delirium.' J Fam Pract 1996;43(3):271-3.

[12] Xie C, Xu S, Ding F, et al. Clinical Features of Severe Wasp sting patients with dominantly toxic reaction: analysis of 1091 cases. PLoS One 2013;8(12): e83164.

[13] Tsai CL, Fang CC, Chen WJ, et al. Hornet sting-induced toxic hepatitis. Clinical Toxicology (Phila) 2005;43(2):127-8.

[14] Barr-Nea L, Neuman MG, Eschr J, et al. Histopathological changes in rat liver following repeated vespine envenomation. Liver 1985;5(6):32635 .

[15] Neuman MG, Eshchar J, Cotariu D, et al. Hepatotoxicity of hornet's venom sac extract in isolated perfused rat liver. Acta Pharmacol Toxicol (Copenh) 1985;56(2):133-8.

[16] Kumar V, Nada R, Kumar S, et al. Acute kidney injury due to acute cortical necrosis following a single wasp sting. Renal Failure 2013;35(1):170-2.

[17] Dhanapriya J, Dineshkumar T, Sakthirajan R, et al. Wasp sting-induced acute kidney injury. Clinical Kidney Journal 2016;9(2):201-4.

[18] Sharma M, Das HJ, Barman AK, et al. Acute kidney injury due to multiple wasp stings. Saudi J Kidney Dis Transpl 2017;28(1):196-8.

[19] Sharmila RR, Chetan G, Narayanan P, et al. Multiple organ dysfunction syndrome following single wasp sting. Indian J Pediatr 2007;74(12) :1111-2.

[20] Ittyachen AM, Abdulla S, Anwarsha AF, et al. Multiorgan dysfunction secondary to severe wasp envenomation. International Journal of Emergency Medicine 2015;8:6. 